\title{
Reverse pumping: theory and experimental validation on a multi-kites system
}

\author{
Rogelio LOZANO Jr, Jonathan DUMON, Ahmad HABLY \\ Gipsa-lab, 11 rue des Mathématiques, \\ Grenoble, France \\ rogelio.lozano, jonathan.dumon, ahmad.hably, @gipsa-lab.fr
}

\begin{abstract}
Most kite wind power systems have a great drawback that wind turbines do not have: they cannot stay in the air if the wind is not strong enough, [7-16]. As a consequence, most of the kite systems need to land when there is no wind, and to take-off once the wind is strong enough. These maneuvers are quite risky because generally the wind gets weak and turbulent close to the ground's surface. Moreover, as the wind can be strong enough at high altitude and weak close to the ground, it might lead to losses in energy production. From a material point of view, "classic" landings and takeoffs need a landing zone, ground handling or infrastructure (such as pylons) that reduces the advantages of kite systems. Some ideas, such as embedded motors or helium balloons, might solve this problem, but they have their own drawbacks such as the weight of the motor and its battery, the necessity of a conductive cable or the need to refill the balloons.
\end{abstract}

The following paper studies a solution called "reverse pumping". It basically consists of providing kinetic energy to the kite by pulling the kite with a rope. This kinetic energy is then transformed into potential energy by gaining altitude. This technique allows to keep the kite airborne in total absence of wind. This paper will study the reverse pumping principle, the constrains on the aerodynamical model, flight simulations and will present the experimental setup used to validate the theoretical study.

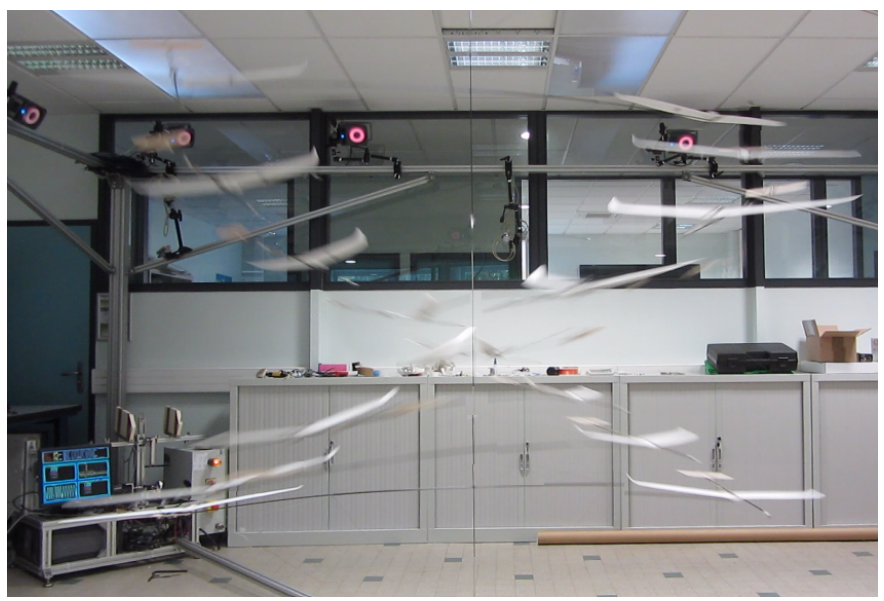

Fig. 1. The experimental protocol in flight.

\section{INTRODUCTION}

The following technique is not intuitive at all. It consists of maintaining the kite in the air, by pulling it with a force directed to the ground. These kinds of phenomena are in fact somewhat common, for example, the classic yo-yo game can use a force directed to the ground to maintain a mean altitude over many up and down cycles that remain constant. In the case of the yo-yo, the force used to push the yo-yo to the ground is then converted in kinetic energy, and is then reused by the yoyo to regain altitude. In the case of the kite system, the basic principle is similar, but the system is more complex as it has various control inputs that can be used to optimize the energy consumption, and it obeys aerodynamic forces. In order to characterize reverse pumping, we have to study the energy consumption and how it changes with respect to the aerodynamic properties of the kite, which altitude can be attained. The following study uses only the tension of the rope to transmit energy to the kite. The main objectives of this paper are :

- To study the influence of the L/D ratio on performance.

- To propose a flight plan for performing reverse pumping.

- To study the cost of maintaining the kite in flight with this technique in order to state whether it is more profitable to use this reverse pumping technique.

The study is composed of a theoretical investigation of reverse pumping, numerical simulations applied to a twin kites system and finally, validation of our simulations on our experimental setup. The paper is organized as follows; Section II introduces the studied system, Section III presents the aerodynamical model. The description of the principle of reverse pumping is described in Section IV and the performances are described in Section V. The control algorithm will be described in Section VI, the experimental protocol in Section VII and the results will be described in Section VIII.

\section{STUDIED MODEL}

This study would be incomplete without an experimental validation. As a consequence, we need to study a model that we can build, with a position which can be measured and controlled properly. It is a difficult task that is unfortunately often skipped, as simulations tend to be presented as a proof of concept.

\section{A. Guidelines}

Taking into account the restrictions due to the construction of a working prototype, the model is designed using the following guidelines:

1) The model's state will be measured using a Vicon(C) optical tracking system. Therefore, it needs to be able 
to perform reverse pumping flying in a space of $3 \times 3 \times 3$ meters, which is the flight domain in which the Vicon system can perform the measurement.

2) Aerodynamical forces obey to equations that become very complex if they are not studied in the correct domain. We will try to reduce the error due to the model as much as we can. Therefore, the shapes of the kite will be simplified, compared to standard kites, but it will greatly enhance the accuracy of the model and therefore the study's relevance.

3) For simplification, we will only control the inputs that are necessary to perform reverse pumping, i.e. the angle of attack and the rope's length. Many freedom axes will be locked as the wings will be fixed.

As a result of these guidelines, an experimental protocol was built, which corresponds to a simplified model of the twin kites [18]. The main difference is that the kites have much simpler movements (see Figure 2). The construction of the protocol makes that they can only fly turning symmetrically around a vertical axis, keeping a constant distance from each other. The two inputs are the pitch angle control $U_{\alpha}$, which is the same for the two kites and the control of the main rope's length $U_{r}$.

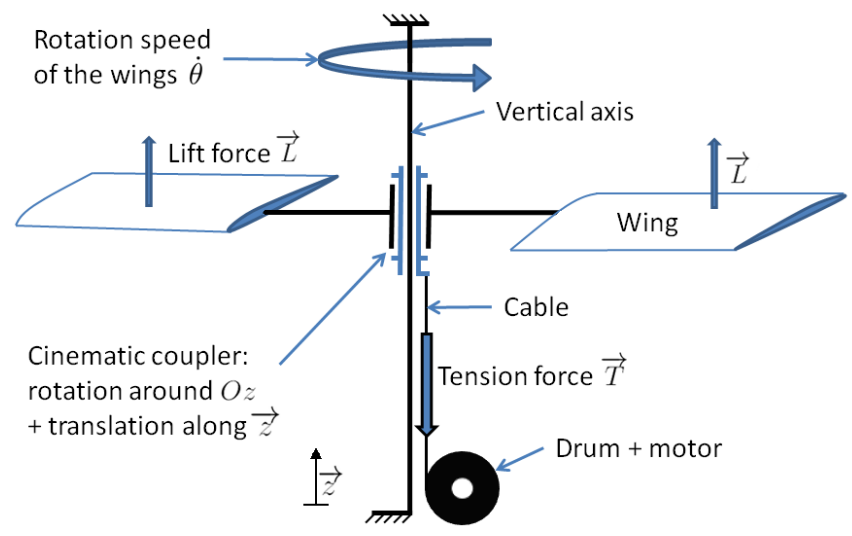

Fig. 2. Scheme of the experimental setup.

\section{B. Inspiration}

The kite system has been inspired by the Otto Lilienthal's 'Whirling arm', (see Figure 3). It was probably the first aerodynamic forces measurement machine [1].

Using the knowledge brought by this machine, Lilienthal built many different kinds of gliders that made successful short flights. He died in 1896, when a wind gust made him lose the control of his machine. He is now considered the father of Aeronautics. One hundred and twenty years later, Lilienthal's sense of simplification and his experimental techniques are still used for experimental aeronautical research and has led to major innovations [2-4].

\section{AERODYNAMICAL MODEL}

Standard kites can be studied with the classic airplanes equations of flight mechanics. Our system is a bit different

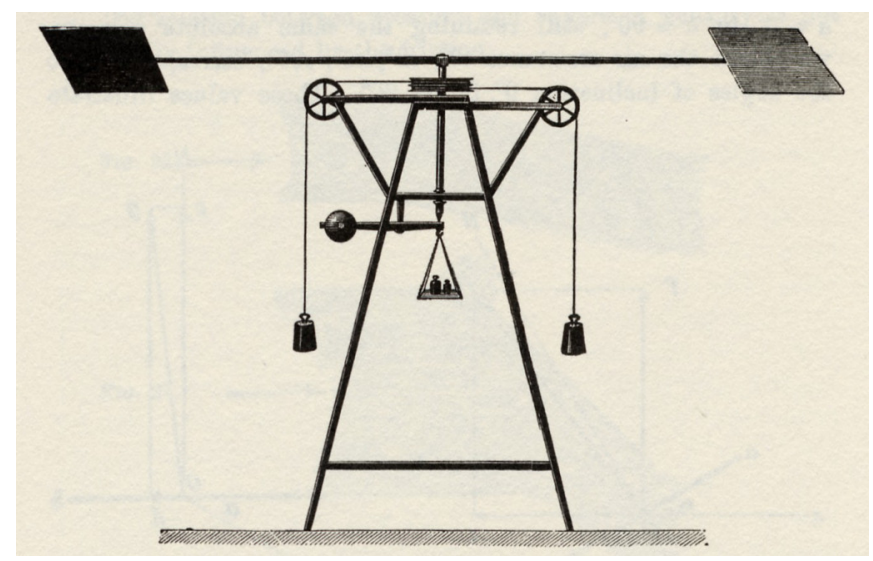

Fig. 3. The Lilienthal's 'Whirling arm'.

because as the kites fly in small circles, they pass through their own downwash and generate a wake that has a vertical speed $V_{w}$, that can be described by the basic equations of a hovering helicopter. This wake's speed tends to greatly diminish the performance of our system, but it will probably not exist in outdoor models, as the kites pass less quickly by the same point. In this case, they can be studied using the standard flight mechanics equations. Other phenomena make it complex to study, like the non constant wind speed along the wingspan, the strong ground effect, etc... In order to reduce the model's errors, the first study will be done with the helicopter equations to study how to design the experimental setup so that it can be studied with the classic airplane's flight dynamics equations. The experimental setup will be designed using the knowledge brought by helicopter theoretical equations.

\section{A. Helicopter Theory}

By studying helicopter theory equations [6], one can see how design parameters act on the system. As the two wings turn around an axis, they define a rotor disc that generates lift and a wake (see Figure 4). From this point of view, the system is similar to the rotor disk defined in standard helicopter theory, however there are still some differences between the initial assumptions of helicopter theory and the our experimental setup: the blade's length are smaller than the radius of the disc, the system flies in presence of ground effect and some "roof effect", the rotation speed of the rotor is low. The wake generated by the downwash reduces the efficiency of the system because it changes the angle of relative wind speed and makes the lift turn backwards. As a consequence, the torque generated by the lift decreases as well as the general efficiency of the system.

The following results are extracted from Helicopter Theory, by Jahson [6]. Considering a rotor disc that generates a lift force $L$, has a rotor disc area $A$, an air density $\rho$, constant climbing speed $V_{c}$ along the vertical axis $\vec{Z}$, the speed $V_{w}$ of the wake that passes through the rotor disc is given by (1):

$$
V_{w}=-\frac{V_{c}}{2}+\sqrt{\left(\frac{V_{c}}{2}\right)^{2}+\frac{L}{2 \rho A}}
$$

Using the mean values of the lift over a cycle, we can get an approximate idea of what the value of the wake will be. 


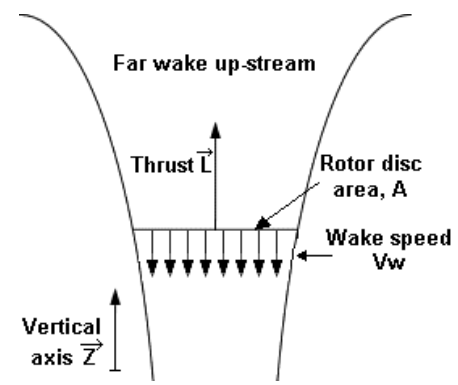

Fig. 4. Side view of the rotor disc and it's wake.

Additionally, ground effect tends to diminish $V_{w}$ by a factor $K_{G}=1-(R / 4 z)^{2}$, where $R$ is the rotor's radius and $z$ it's height. Note that in order to remain in flight, the lift force $L$ needs to be greater or equal to the weight of the prototype. As a conclusion, in order to reduce the value of downwash, one needs to build a prototype that is very light, and at the same time, has a large diameter to increase the disc area $A$. These two characteristics are obviously hard to conciliate.

\section{B. Flight Mechanics Approach}

A standard flight mechanics approach to the problem will be done to try to model the behavior of the kite. The results will be then compared to experimental results. In this section, the wake $V_{w}$ is assumed to equal zero. The trajectories and the study of the system will be calculated taking as a basis the following equations. The forces applied to each wing wing are described in Figure 5. Note that the aerodynamical forces acting on both wing have the same strength. For the sake of representation, half of the traction force $T / 2$ and half of the weight $W / 2$ are sketched in Figure 5, permitting us to draw the acceleration, lift and drag vectors corresponding to only one of the two wings. The total lift force is $2 L$, total drag is $2 D$ and acceleration rate is $2 a$. The equations of lift $\vec{L}$ and

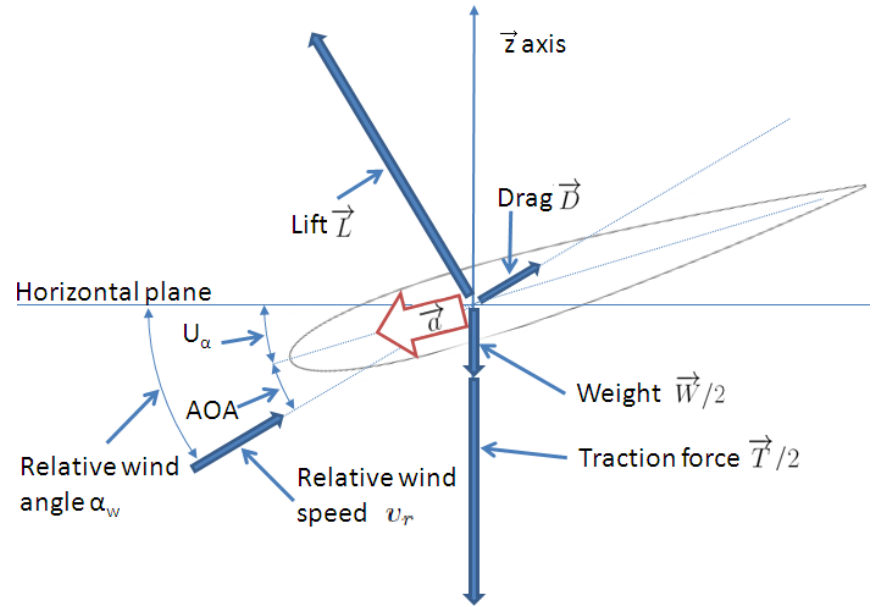

Fig. 5. Forces diagram for each wing.

drag $\vec{D}$ are defined for each wing using standard notation, i.e. the drag is directed in the same direction as the relative wing angle, and the lift applies orthogonal to it, in the direction of positive angle of attack $(\mathrm{AOA}=\alpha)$ as depicted in Figure 5.
The strength of each force is described in (2),

$$
L=\frac{1}{2} \rho S v_{r}^{2} C_{L} \quad ; \quad D=\frac{1}{2} \rho S v_{r}^{2} C_{D}
$$

where $\rho$ is the air density, $S$ is the surface of each wing, $v_{r}$ is the relative wind speed and the lift coefficient is $C_{L}$ (3) and the drag coefficient $C_{D}$ (4) are:

$$
\begin{gathered}
C_{L}=\frac{\partial C_{L}}{\partial \alpha}\left(\alpha_{w}+U_{\alpha}\right)+C_{L 0} \\
C_{D}=\frac{C_{L}^{2}}{\pi e \lambda}+C_{D 0}
\end{gathered}
$$

where $C_{L 0}, \lambda$ is the aspect ratio and $U_{\alpha}$ is the pitch angle. The zero lift drag coefficient $C_{D 0}$ will take into account the drag of the structure. In this case, Oswald's efficiency factor $e$ should not be defined as it usually is because the distribution of lift along the chord is not symmetric [5]. This $e$ factor is normally used to calculate the AOA that leads to the best L/D ratio but in this case, we will not use $e$ and directly work with the $\mathrm{L} / \mathrm{D}$ ratio. Also, note that when the kite is performing a non accelerated flight at a constant $r \dot{\theta}$ with $T=0$, its corresponding glide slope is $G S(\alpha)=-\arctan C_{D} / C_{L}$. At that moment, the sum of the lift and drag vectors is vertical, compensates the weight of the kite, and the kite's acceleration is equal to zero. This gliding slope $G S(\alpha)$ is an important parameter because it defines the minimal gliding slope needed in order to perform reverse pumping. The classic way to reduce the value of this gliding slope $G S(\alpha)$ is to make $\lambda$ greater. The second way is to fly with the optimal AOA, which in this case will more complicated as it will be used for control purposes.

\section{Relative Wind Speed}

Relative wind characteristics will be defined, assuming the air is still. The relative wind speed will be approximated by the sum of the horizontal speed $v_{h}$ and the vertical speed $v_{v}$ (5).

$$
v_{h}=\dot{\theta} r \quad ; \quad v_{v}=\dot{z}+V_{w}
$$

In the equation (5), the radius $r$ is the distance between the rotation axis and the middle of the wing. Note that in this case, the horizontal windspeed $v_{h}$ is an approximation because it is not constant along wingspan. In our experiment, for each wing, the relative wind speed difference from the right wingtip to the left wingtip is approximatively of 4 . If the radius $r$ would be much greater than the wingspan, the horizontal windspeed $v_{h}$ over one wing could be approximated as constant and equal to $r \dot{\theta}$, and one could use the $e$ factor and the equation 4 to calculate the $\mathrm{L} / \mathrm{D}$ ratio.

Using (5) we obtain the wind angle $\alpha_{w}$ (6), which is the angle of the wind velocity vector measured with respect to the horizon. The wing's relative wind velocity is given by $v_{r}(7)$.

$$
\begin{gathered}
\alpha_{w}=-\arctan \frac{\dot{z}+V_{w}}{\dot{\theta} r} \\
v_{r}=\sqrt{(\dot{\theta} r)^{2}+\left(\dot{z}+V_{w}\right)^{2}}
\end{gathered}
$$

Note that when $V_{w}=0$, the gliding slope $G S$ is defined by the relative wind angle $\alpha_{w}=-\arctan \frac{\dot{z}}{\dot{\theta} r}$. 


\section{Acceleration Rates}

This system can have two kinds of accelerations: vertical and rotational. The vertical acceleration $\ddot{z}$ is given by (8):

$$
\ddot{z}=\frac{L \cos \left(\alpha_{w}\right)+D \sin \left(\alpha_{w}\right)-w-T}{m}
$$

The rotational acceleration $\ddot{\theta}$ is given by (9):

$$
\ddot{\theta}=2 \frac{r a}{J}=2 \frac{r L\left(\sin \alpha_{w}\right)-r D\left(\cos \left(\alpha_{w}\right)\right.}{J},
$$

where $J$ is the moment of inertia. Its value will be approximated by $J=m r^{2}$, where $\mathrm{m}$ is the mass of the system. These two equations will be used to study the model's performances and behaviors depending on the $L / D$ ratio and on the gliding slope.

\section{BASIC PRINCIPLE of REVERSE Pumping}

The basic principle of reverse pumping lies in the exchange of energy during two different phases: the "kinetic energy charge" phase and the "potential energy transfer" phase. Kinetic energy charge happens between the time $t_{0}$ and $t_{1}$, when the kite will convert the energy provided by the rope's tension into the kite's kinetic energy. Potential energy transfer happens from the time $t_{1}$ to $t_{2}$, when the kite transforms the kinetic energy into potential energy by acquiring height. We differentiate four different kinds of energy. The energetic cost $\Delta E_{t}$ is the energy brought by the rope's tension $T$. Its value can be written as:

$$
\Delta E_{t t_{0} \rightarrow t_{1}}=\int_{t_{0}}^{t_{1}} T \dot{z} d t .
$$

The variation kinetic energy of the kite is:

$$
\Delta E_{c t_{i} \rightarrow t_{j}}=1 / 2 m\left(V_{j}^{2}-V_{i}^{2}\right),
$$

and the variation potential energy is (12):

$$
\Delta E_{p t_{i} \rightarrow t_{j}}=m g\left(z_{j}-z_{i}\right) .
$$

During the kinetic transfer phase phase, $i=0$ and $j=1$, and during the potential transfer phase, $i=1$ and $j=2$. These energy variations obey the following equations:

$$
\Delta E_{t}=\Delta E_{c}+\Delta E_{p}+\Delta E_{\text {lost }}
$$

where $\Delta E_{\text {lostt }_{i} \rightarrow t_{j}}$ is the loss of energy due to drag.

There are several ways to transmit energy during the kinetic energy phase, such as using a constant tension $T$, a constant slope, or changing angles of attack. This study will focus on a technique using constant slope and constant angle of attack. Its main advantage is simply that its simpler to explain and study. For the potential energy transfer, the study will consider flight with a constant angle of attack. Note that the novelty of the study lies on the first phase.

\section{A. Initial Conditions}

The initial conditions correspond to a non accelerated flight of the kite with rope tension $T$ equal to zero. The initial speed $V_{0}$ is chosen such that the sum of the lift and drag vectors compensates the weight vector $m g$, and therefore $V_{0}=\sqrt{\frac{2 m g \cos G S(\alpha)}{\rho S C_{L}}}$. The angle of attack is kept constant, and the corresponding gliding slope is $G S(\alpha)$. At that instant, the kite's acceleration is equal to zero and it behaves like a glider in non accelerated flight. The initial height is greater than $z_{0}$.

\section{B. Kinetic Energy Charge Phase}

The kinetic charge phase starts when the glider's height is equal to $z_{0}$. A tension $T$ will force the system to follow a straight trajectory with a forced slope where the value will be less than the gliding slope $G S(\alpha)$. The forced gliding slope is defined as $F S=G S(\alpha)-\Delta_{G S}$, where $\Delta_{G S}$ is the slope added by pulling the kite. As a consequence, the sum of $L$ and $D$ will no longer be aligned with the weight and generate an acceleration vector that will increase $r \dot{\theta}$ and therefore the kite's kinetic energy. The horizontal component of the acceleration vector is the one part that increases the horizontal speed $r \dot{\theta}$ and its acceleration is given by (9). The end of this period $t_{1}$ happens when the kite reaches its lowest height $z_{1}$, while respecting its maximum speed $V_{\max }$. If this phase is effective, the kite's energy $\Delta E_{c}+\Delta E_{p}$ is greater at $t_{1}$ than at the initial time $t_{0}$.

\section{Potential Energy Transfer Phase}

At the beginning of the potential energy transfer phase $t_{1}$, the potential energy of the kite is $E_{p}\left(t_{1}\right)=m g z_{1}$. During this phase, the transmitted kinetic energy $\Delta E_{c t_{0} \rightarrow t_{1}}$ will be transformed into potential energy by gaining altitude. The tension $T$ of the cable is set to zero during the entire phase. The kite will transform its kinetic energy into potential energy by gaining altitude until the kites reaches it's maximal height or it's speed goes below the initial speed $V_{0}$. This flight can be done with different flight plans. The two principals are flying with a constant AOA, of flying with a constant gliding slope, that is controlled by acting on the AOA. The end of this phase happens when the kite has regained its original flight speed $V_{0}$. The reverse pumping is effective if the height $z_{2}$ is greater or equal to $z_{0}$.

\section{Standard flight plan}

To sum up, a classic flight plan looks like Figure 6. In this particular case, the end of the potential transfer phase happens when the kinetic energy reached its initial value, and the final height is much greater than the initial value.

\section{PERFORMANCES OF THE SYSTEM}

In this study, three different characteristics will be analyzed and we will try to study their relation with the L/D ratio. The most important factor is $\xi$, the variation of kinetic energy per loss of altitude during the kinetic charge phase, given by equation (14). Its value must be greater than one to make the reverse pumping possible. One can see in Figure 7 that $\xi$ increases with the $L / D$ ratio and the $\Delta_{G S}$ value. Note that 

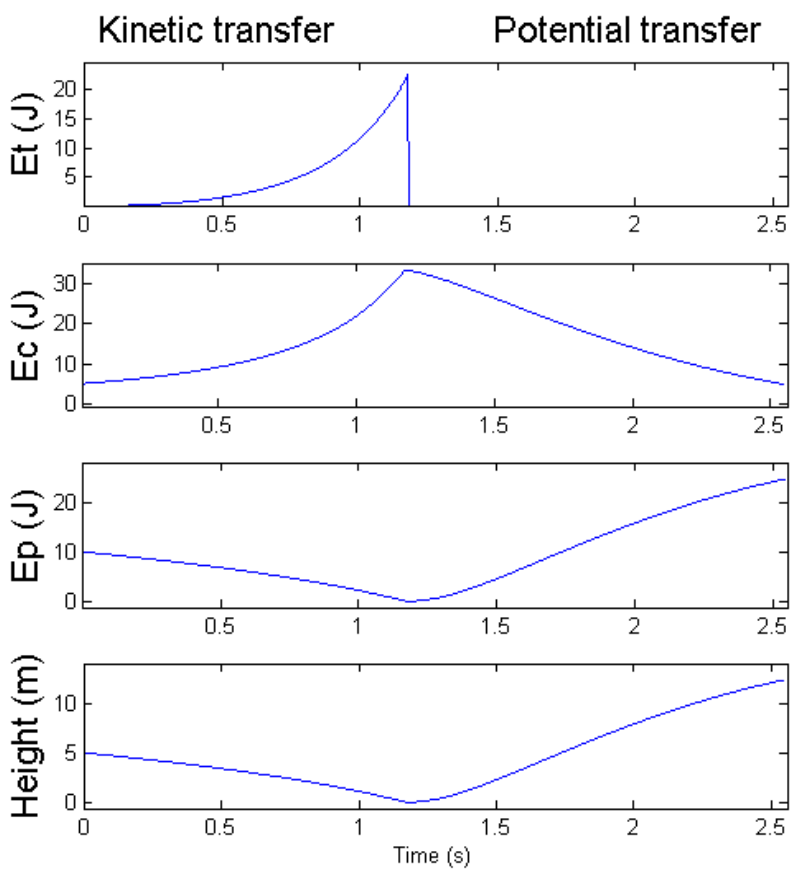

Fig. 6. Energy variations during the kinetic charge phase and the potential transfer phase. Note that the final height is greater than its initial value. ( L/D $=20, \Delta_{G S}=20$ degrees)

if $\Delta_{G S}=0, \xi$ is necessarily equal to zero and the reverse pumping is not possible.

$$
\xi=-\frac{\Delta E_{c t_{0} \rightarrow t_{1}}}{\Delta E_{p t_{0} \rightarrow t_{1}}}
$$

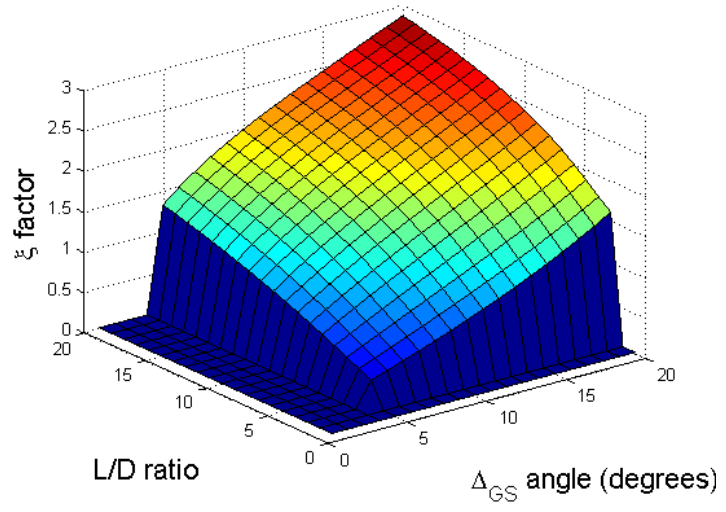

Fig. 7. Variation of kinetic energy divided by the lost potential energy during the kinetic charge phase.

The factor $\eta_{1}$ characterizes the efficiency of the transfer during the kinetic charge phase. Its expression is given by equation (15) and plotted in Figure 8. Note that this parameter gives information about how much it will cost to make a kite stay in the air. The cost worthiness depends of many other parameters that are quite hard to estimate, like the risk of crashing, how long the wind speed is going to be low, etc.

$$
\eta_{1}=-\frac{\Delta E_{p t_{0} \rightarrow t_{1}}+\Delta E_{c t_{0} \rightarrow t_{1}}}{\Delta E_{t t_{0} \rightarrow t_{1}}}
$$

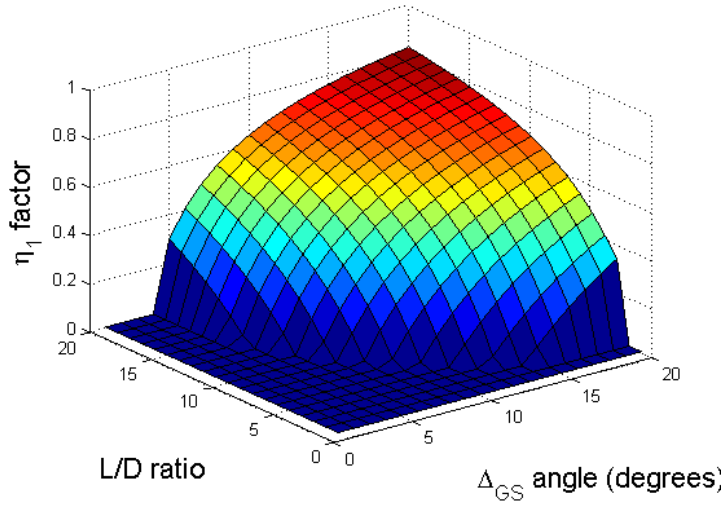

Fig. 8. Efficiency of the kinetic charge phase.

The $\eta_{2}$ parameter characterizes the efficiency of the potential transfer phase, i.e. the efficiency of the energy transfer from the gain kinetic energy $E_{c}$ to the potential energy $E_{p}$, see equation 16. As the L/D ratio increases, $\eta_{2}$ gets closer to 1, see Figure9.

$$
\eta_{2}=-\frac{\Delta E_{p t_{1} \rightarrow t_{2}}}{\Delta E_{c t_{1} \rightarrow t_{2}}}
$$

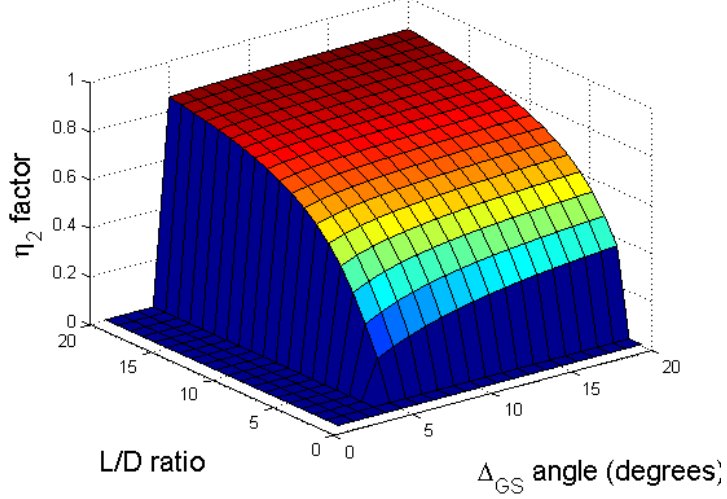

Fig. 9. Efficiency of the potential transfer phase.

The reverse pumping increase of energy per cycle can be characterized by the energy gain $\xi \eta_{2}$ value plotted in Figure 10. Note that in order to be effective, $\xi \eta_{2}$ has to be greater or equal to 1 , the design of the flight plan and the kite's characteristics must respect this condition. One can see that in this case, many combinations of low values of $L / D$ and $\Delta_{G S}$ cannot satisfy the requirements of reverse pumping.

Note two limits are not taken into account in the previous plots: the fact that the motor that pulls the kite has limits on its performances and the maximum load of the kite system. As this study is made for a constant angle of attack, the max speed has a limit that can be calculated using the maximal load of the kite and equation (2). 


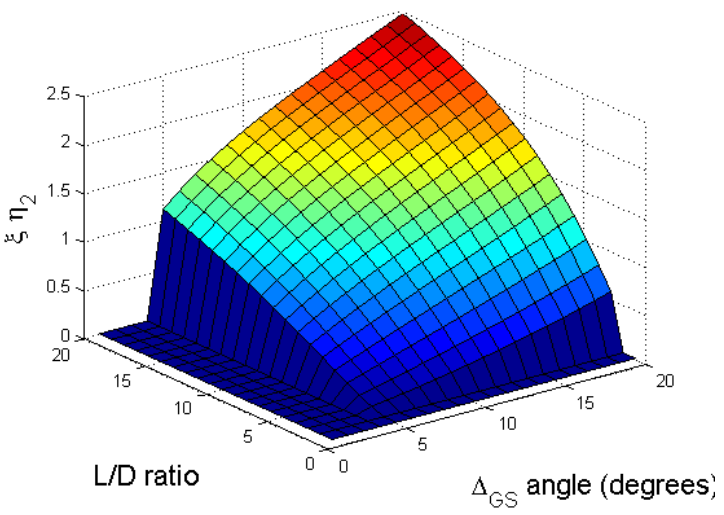

Fig. 10. Increase of energy per cycle. Note that it increases with the $L / D$ ratio and $\Delta_{G S}$

\section{A. Cost of flight time}

In order to know if it is worth using the reverse pumping technique, one has to compute its cost, and compare it to the cost of making the kite land and then takeoff that depends on the kite type. This section will present an approximated formula in order to compute the cost of the flight time.

During a cycle, the consumed energy is $\Delta E_{t}$. The end of the potential transfer phase can be defined as the moment when the kite's speed is $V_{0}$ and that all the maximum height has been attained. In this case, the maximal height value is $z_{\max }=z_{0} \xi \eta_{2}$. The height between $z_{0}$ and $z_{\max }$ can be spend by the kite gliding until it starts another cycle of kinetic charge phase. The flight time FT will be approximated by the gliding time, neglecting the kinetic charge and potential transfer phases lengths. Like the initial condition, the flight speed is $V_{0}$ and the gliding slope being $G S(\alpha)$, the vertical speed is then $V_{z}=$ $V_{0} \sin G S(\alpha)$. The cost of the flight time in Watts will be named $X$, and is given by equation (17):

$$
X=\frac{\Delta E_{t}}{F T} \quad ; \quad F T=\frac{z_{0} \xi \eta_{2}-z_{0}}{V_{0} \sin G S(\alpha)}
$$

In this special case, for values of $L / D=20$ and $\Delta_{G S}=20$ the cost of the flight time is approximately $1.8 \mathrm{~W}$. A standard flight with an embedded propulsion system like a propeller would consume approximately $0.5 \mathrm{~W}$, see [5].

\section{CONTROL OF THE SYSTEM}

\section{A. Control architecture}

The system is composed of 4 main parts. The kite system includes the two kites with an embedded control unit that controls the tail position so that the angle of attack of the wing takes the desired position. The position is then measured with a Vicon motion capture system and sent to a real time computer that makes the control loop and transmits the orders to the ground station. The ground station has a motor and a controller used to pull the kites down with the desired position.

\section{B. The angle of attack control}

The angle of attack of the kite can be controlled with different techniques. Some experiments have used the length of the cable or their attachment point to control it. This experiment will use a method that is classically used in airplanes; a tail has been mounted on the wing to control it. The major advantage of this method is that the angle of attack has a stable value that depends on the angle of attack of the tail [5]. Normally, a PID controller is enough to reject perturbations and allows proper control of the AOA. The main use is to reduce oscillations, the $\mathrm{D}$ parameter is then the most important. In this particular case, the control could not be implemented because of the short length of each cycle. The total response time, from acquisition of the position to the stabilization of the AOA could not be done within the duration of a the kinetic charge phase, as its duration is approximately $0.2-0.5$ seconds. The passive stability of the system was used, but led to an approximate control of the AOA.

\section{The altitude control}

A rope and a servo motor are used so that the kite follows the forced gliding slope $F S$ during the kinetic transfer phase. The value of $F S$ is controlled by $F S=G S(\alpha)-\Delta_{G S}$ and GS is approximated by $-\arctan \frac{\dot{z}}{\dot{\theta} r}$. The $\dot{\theta} r$ value is controlled by aerodynamic forces and the value of $\dot{z}$ is computed so that it follows $-r \dot{\theta} \tan (F S)$. To do so, a PID controller has been implemented to control the motor's position. More complex algorithms such as LQR could be used to diminish the consumption of the motor. The reduction of consumption comes as a more complex work that does not seem relevant before the reverse pumping functions properly.

\section{EXPERIMENTAL SETUP}

A movie showing the experimental results can be viewed at http://www.gipsa-lab.grenoble-inp.fr/recherche /plates-formes.php?id plateforme $=70$

\section{A. Previous experiments}

Our first tests were made on the Satellite I and Satellite II prototypes. The results showed that there was an acceleration due to the kinetic transfer but the experimental setup were too complex to measure and to control properly all the axes.

\section{B. The kites}

This error was solved by using a simpler model where one only has to focus on the reverse pumping and much less on axis stabilization. The early version of the proposed experimental prototype had an angle of attack that was directly controlled by a servo fixed to the carbon tube. This led to a low accuracy on the AOA control because the main carbon tube had the tendency to twist over the longitudinal axis. Of course, the system had to be light, so the chose has been made to use auto stabilized wings, the tube does not need anymore to have a axial stiffness. The control of the AOA got enhanced, but the stabilization system would need to have lower response times in order to be really effective.

The kites wings are built with a mix of EPP foam and a carbon fiber tube that is used to hold the tail (see Figure 12). The most important characteristic of this wing is that its aspect ratio is 10 . The tail is a piece of balsa wood controlled by the embedded control unit AR6400 SPECTRUM receiver 


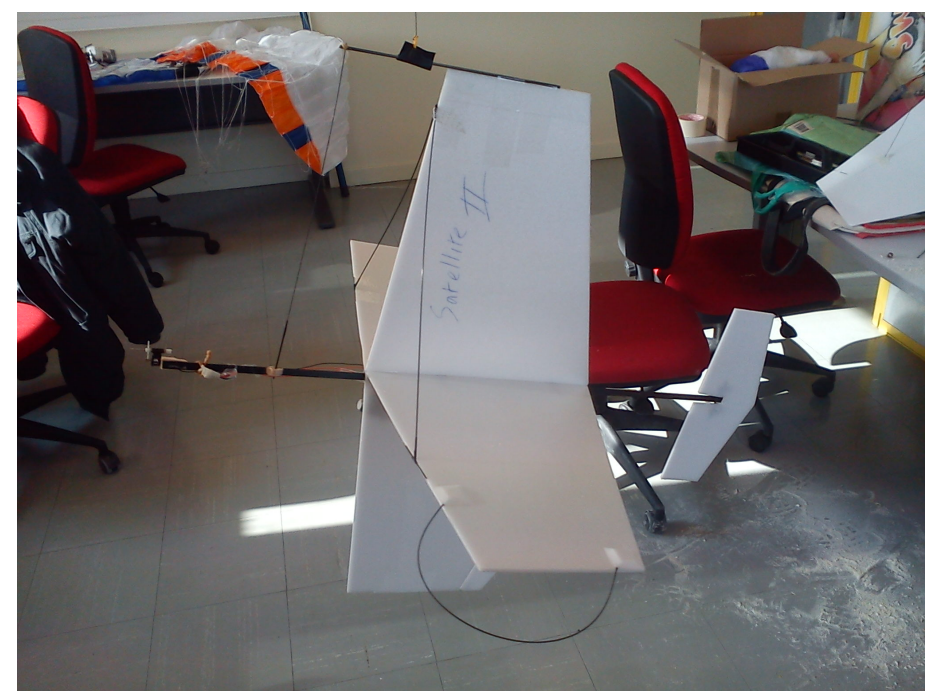

Fig. 11. The Satellite 2 prototype. The horizontal wing was used to generate the lift used to counter weight, and the vertical wing was used to produce a lift force for accelerating the kite.

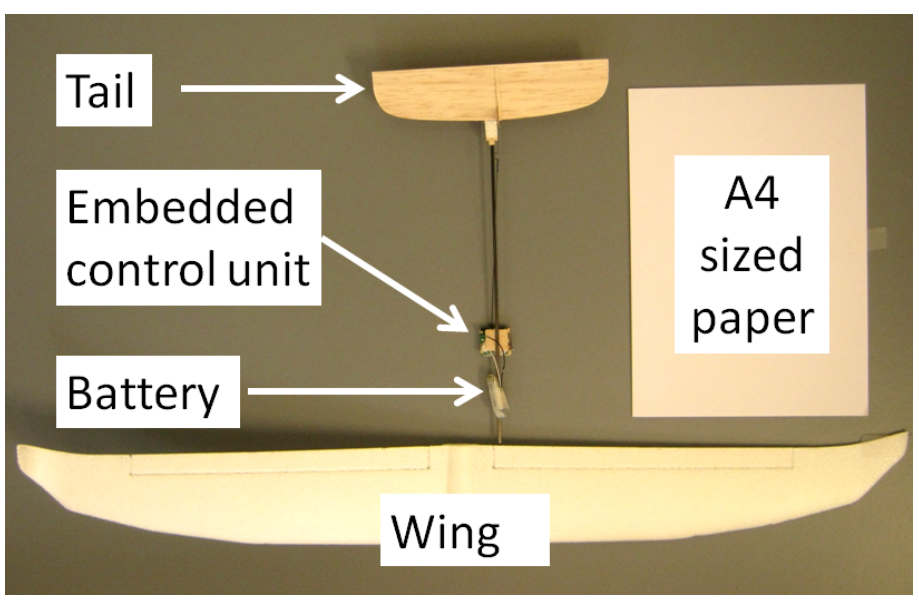

Fig. 12. The wing.

that has two build-in linear servos. The total weight of each wing is 40 grams. Having a low weight allows to have very low flight speeds, which is a very important characteristic. On the other hand, light structures are less resistant to shocks and to aerodynamic forces. These wings showed us that they start twisting as the wind speed reaches $8 \mathrm{~m} / \mathrm{s}$ and their properties change a lot in these conditions.

\section{System's characteristics.}

The built prototype has the following characteristics, see Table I:

\section{EXPERIMENTAL RESULTS}

\section{A. Experiments vs simulations}

The first flight tests had the objective to determine the L/D ratio. The wings that were used usually have a L/D ratio that can go up to 20 . The tests revealed that the whole system could not have a $\mathrm{L} / \mathrm{D}$ ratio greater than 5.5. The main reasons are that
TABLE I. COEFFICIENTS OF THE EXPERIMENTAL SET-UP

\begin{tabular}{lll}
\hline Symbol & Name & Value \\
$M$ & mass & $0.15 \mathrm{Kg}$ \\
$r$ & radius & $1.2 \mathrm{~m}$ \\
$\rho$ & air density & $1.225 \mathrm{Kg} / \mathrm{m}^{3}$ \\
$S$ & wing area (each) & $0.065 \mathrm{~m}^{2}$ \\
$e$ & Theoretical $e$ factor & 0.9 \\
$\lambda$ & aspect ratio & 10 \\
$\frac{\partial C L}{\partial \alpha}$ & lift derivative w.r.t. AOA & $0.05 \mathrm{deg}-1$ \\
$C_{D 0}$ & zero lift drag & 0.01 \\
$C_{L}$ & lift coefficient at AOA $=0$ & 0 \\
$L / D$ & L/D (simulations) & 3 to 20 \\
$L / D$ & L/D (measured) & 5.5 \\
$G S$ & gliding slope (measured) & $10 \mathrm{degrees}$ \\
$V_{0}$ & initial air speed & $6 \mathrm{~m} / \mathrm{s}$ \\
$V_{\max }$ & max speed & $8.5 \mathrm{~m} / \mathrm{s}$ \\
$z_{0}$ & initial altitude & $1.5 \mathrm{~m}$ \\
$z_{1}$ & min altitude threshold & $0 \mathrm{~m}$ \\
\hline
\end{tabular}

the wind speed along the wing is not constant, which has an effect equivalent to reducing the value of the $e$ parameter. The second reason is due to drag and friction forces; the carbon structure that unites the two wings seems to be a great source of drag, although it is made out of a carbon tube of only $4 \mathrm{~mm}$ of diameter. Friction forces also made the L/D ratio go low, even using ball bearings in every single rotating part. The low value of L/D ratio made the tests much more complex than expected as it led to great losses of energy.

The second difference is that the wings had a tendency to twist as aerodynamic forces got too strong. It has been noticed that high frequency oscillations appear in the wing tips, which made the AOA have great variations, instead of staying constant at 10 degrees.

The last great difference is that the displacement over $z$ has a range of only 1.5 meters, whereas it has a value of 5 meters in the simulations presented in previous section. As a consequence, the system can not reach high values of $\xi$ that are needed to perform reverse pumping. The acceleration $\ddot{z}$ also had the tendency of generating big variations on the AOA value. Depending of the value of the acceleration, it could generate oscillations that would increase the AOA, making greater the acceleration of the system, and leading to some results greater that what was expected, see Table II.

\section{B. The kinetic charge phase}

As a consequence of the low $\mathrm{L} / \mathrm{D}$ ratio and of various friction forces, only the kinetic charge phase could be studied. The Table (II) gives the result for the $\xi$ values depending on the $\Delta_{G S}$ values.

The first thing that must be seen is that this experimental setup led to a max value of $\xi=2.72$. This means that reverse pumping is possible as long as the kite system can have a $\eta_{2}$ value that is greater than 0.36 , in order to satisfy $\eta \xi_{2} \geq 1$. One can see in Figure 10 that this value of $\eta_{2}$ can hardly be reached with a $\mathrm{L} / \mathrm{D}$ ratio of 6 . Adding the friction due to the the kinematic coupler (see Figure 2) and the approximate control of the AOA, the needed $\eta_{2}$ can not be reached. Note that the mean error ratio between the measurements of $\xi$ and the simulations goes from 0.37 to 1.45 . The first thing that comes to mind is that the level of precision is not very high. The general lack of accuracy on the control of the AOA can be seen as the main reason for the unexpected results. The $\xi$ 
values corresponding to $\Delta_{G S}=30$ and 35 degrees are greater than theory because of the bad control of the AOA, instead of having a value of 10 degrees, their value was measured with peaks up to 25 degrees, which generate an acceleration that explains such values of $\xi$.

TABLE II. RESULTS FOR THE $\xi$ PARAMETER

\begin{tabular}{lll}
\hline $\begin{array}{l}\text { Added glide slope } \Delta_{G S} \\
\text { (degrees) }\end{array}$ & Theoretical $\xi$ value & Measured $\xi$ value \\
10 & 0.69 & 0.26 \\
15 & 0.91 & 0.41 \\
20 & 1.11 & 0.49 \\
25 & 1.32 & 0.92 \\
30 & 1.56 & 1.86 \\
35 & 1.87 & 2.72 \\
\hline
\end{tabular}

Figure 13 shows the kinetic and potential energy variations for $\Delta_{G S}$ of 25 degrees. One can see that the beginning and the end of the kinetic charge phase do not have the desired trajectory. The trajectories need to be smoothed in order to avoid mechanical shocks that would harm the structure.
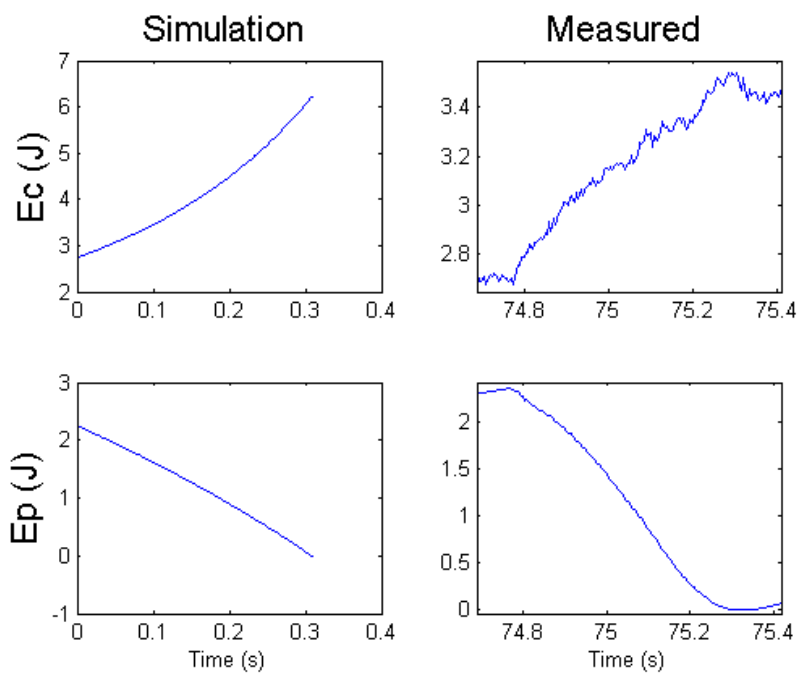

Fig. 13. Comparison between the theoretical results and te measured results. The difference between the two values of $E_{c}$ led to a percentage of error of $40 \%$.

\section{COnclusions}

This paper shows that the reverse pumping is possible as long as the L/D ratio and a max speed are great enough. The kite system does not have stability and aeroelasticity problems. One of its important knowledge brought by this paper is that theoretical and practical work can give quite different results when the practical part has problems. It reminds us the importance of developing simple prototypes that are as close as possible to the theoretical model, or the importance of studying accurate models. The paper provides general information that could be seen as a basis for more detailed studies. Every different phase can have many ways of being optimized, the most important and innovative part seems to be the kinetic transfer phase. Future work shall be done on more performant kite systems.

\section{REFERENCES}

[1] Lilienthal, O. (1911), Birdflight as the basis of aviation.

[2] Don Dwiggins (1979), Man-powered aircraft, Tab Books.

[3] Grosser, M. (2004), Gossamer odysey, The thiumph of Human powered flight, Zenith press.

[4] Conner, M. (2001), Hans Von Ohain: Elegance in flight, American Institute of Aeronautics and Astronautics.

[5] Anderson, J. (2001), Fundamental of aerodynamics, McGraw-Hill.

[6] Johnson, w. (1980), Helicopter theory, Dover publications.

[7] Baayen, J. (2011), Automatic trajectory tracking control of kites, $\mathrm{PhD}$ of Delft University of Technology.

[8] M.Ahmed, A.Hably, and S.Bacha, (2012), High Altitude Wind Power Systems: A Survey on Power Kites, The International conference on electrical machines.

[9] M.Ahmed, A.Hably, and S.Bacha, (2011), Power Maximization of a Closed-orbit Kite Generator System, 50th Conference on Decision and Control and European Control Conference (IEEE CDC-ECC).

[10] M.Canale et al. (2010), High Altitude Wind Energy Generation Using Controlled Power Kites, IEEE Transactions On Control Systems Technology, volume 18(2), pages 279-293.

[11] A.Furey, and I.Harvey, (2007), Evolution of neural networks for active control of tethered airfoils, Advances in Artificial Life Journal, pages 746-755, Springer.

[12] A.Ilzhofer, B.Houska, and M.Diehl, (2007), Nonlinear MPC of kites under varying wind conditions for a new class of large-scale wind power generators, International Journal Of Robust And Nonlinear Control,volume 17, pages 1590-1599.

[13] A.Podgaets, and W.Ockels, (2007), Robust Control of Laddermill Wind Energy System, Proceedings of the Wind Power Shanghai Conference 2007.

[14] C.Novara, and L.Fagiano, and M.Milanese, (2011), Direct data-driven inverse control of a power kite for high altitude wind energy conversion, IEEE International Conference on Control Applications (CCA), pages 240-245.

[15] R.Lozano Jr, J.Dumon, A.Hably, and M.Alamir (2011),Control of a wind power system based on a tethered wing, IFAC EGNCA, Bangalore, India 2011

[16] A.Hably, R.Lozano Jr, and J.Dumon, and M.Alamir, (2011), Observerbased control of a tethered wing wind power system: indoor real-time experiment, To be presented at the 2013 American Control Conference, Washington, USA, 2013 